\title{
Publisher Correction: Dimensionality reduction to maximize prediction generalization capability
}

Takuya Isomura (1) and Taro Toyoizumi (1)

Correction to: Nature Machine Intelligence https:/doi.org/10.1038/s42256-021-00306-1, published online 12 April 2021.

In the version of this Article originally published, in the caption of Fig. 2 the expression $|\theta-\theta|_{\mathrm{F}}^{2} / \max \left(|\theta|_{\mathrm{F}}^{2},|\theta|_{\mathrm{F}}^{2}\right)$ was incorrect; it should have been $|\boldsymbol{\theta}-\theta|_{\mathrm{F}}^{2} / \max \left(|\theta|_{\mathrm{F}}^{2},|\boldsymbol{\theta}|_{\mathrm{F}}^{2}\right)$. In addition, a number of Greek letters were missing or incorrectly formatted in the PDF version of the Article, while the HTML version was correct. This has been corrected.

Published online: 5 May 2021

https://doi.org/10.1038/s42256-021-00352-9

(c) The Author(s), under exclusive licence to Springer Nature Limited 2021 